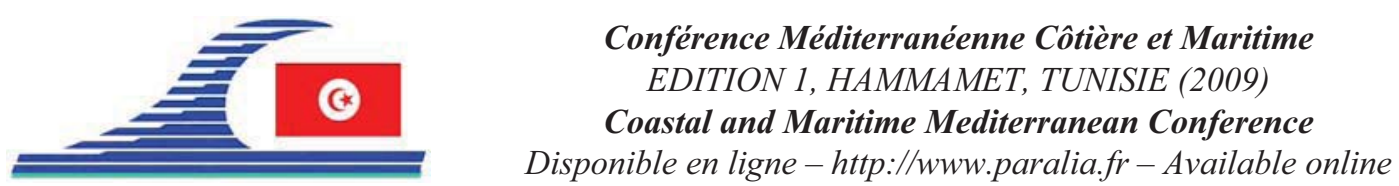

\title{
Mise en œuvre d'une méthodologie pour la valorisation des sédiments de dragage maritime
}

\author{
Mohamed MIRAOUI ${ }^{1,2}$, Rachid ZENTAR ${ }^{2}$, Nor-Edine ABRIAK ${ }^{2}$
}

1. Département Génie Civil \& Environnemental, Ecole des Mines de Douai, 941 rue Charles Bourseul, Douai, France.

2. Université des Sciences et Technologies de Lille U.S.T.L. Cité Scientifique, 59655 Lille, France.

miraoui@ensm-douai.fr

\section{Résumé :}

Les sédiments sont dragués généralement avec une teneur en eau élevée. Donc, une réduction de la quantité d'eau est nécessaire pour optimiser l'étape de traitement ou de stabilisation. Dans ce but, le processus de déshydratation est exploré. Après une détermination des caractéristiques physiques des sédiments (plasticité, teneur en eau ...) et une évaluation des impacts environnementaux, le processus de déshydratation est étudié. A ce stade, les réductions d'eau par infiltration et par évaporation sont mesurées sur la base de deux tests différents.

\section{Mots clés :}

Sédiments de dragage - Techniques routières - Valorisation

\section{Introduction}

Les sédiments de dragage étaient jusqu'alors, soit rejetés en mer ou stockés dans des zones de dépôts à terre. Ces opérations sont extrêmement coûteuses et les services chargés des accès portuaires cherchent le meilleur compromis entre les impératifs du maintien des activités, l'incidence du coût des dragages sur l'économie portuaire et la protection de l'environnement.

Cette étude traite des sédiments du port de Dunkerque-PAD. C'est le troisième port de commerce France en termes de tonnage global. Environ 3 millions $\mathrm{m}^{3}$ sont dragués annuellement au PAD.

\section{Contexte}

L'activité portuaire, maritime ou fluviale, génèrent plus de 50 millions de $\mathrm{m}^{3}$ de sédiments chaque année (ALZIEU, 1999). La législation sur les sédiments (arrêté du 14 juin 2000 paru dans le JO n 184 du 10 août 2000, circulaire du 4 juillet 2008) impose de développer des solutions alternatives pour la gestion durable des matériaux de dragage. Plusieurs études ont démontré la possibilité de réutilisation des sédiments dans 
divers domaines du génie civil (ZENTAR et al., 2008 ; DUBOIS, 2006 ; HAMER \& KARIUS, 2002). Toutefois, beaucoup de travaux de recherche ont concerné l'utilisation des sédiments dans différentes filières de génie civil, telles que : technique routière (DUBOIS, 2006 ; TRAN, 2009), fabrication de briques (HAMER \& KARIUS, 2002), substitution pour fabrication de liants hydrauliques (DALTON et al., 2004). ... etc.

La teneur en eau est élevée et nécessite donc un séchage préalable. Pour cela des essais de déshydratation ont été réalisés pour améliorer les caractéristiques mécaniques du matériau (DUAN, 2008). Nous avons donc, étudié les phases d'infiltration et d'évaporation sur trois séries d'échantillons (figure 1).

\section{Méthodologie}

Dans la présente étude, une caractérisation physique du sédiment a été réalisée (tableau 1) afin de relever les caractéristiques initiales du matériau (granulométrie, perte au feu). Le conditionnement des sédiments et les données relatives au séchage des sédiments sont peu disponibles dans la littérature. Pour optimiser le traitement des stocks de sédiments, la connaissance et le suivi de la diminution de la charge d'eau sont importants. En l'absence d'installation disponible pour la déshydratation des sédiments, on a réalisé une série d'échantillons pour étudier le séchage des sédiments.

Trois diamètres (D) ont été considérés : $35 \mathrm{~mm}, 70 \mathrm{~mm}$ et $105 \mathrm{~mm}$ (voir figure 1).

La hauteur est fixe et égale à $15 \mathrm{~cm}$ pour tous les essais.

Pour ce fait, un protocole expérimental de déshydratation est mis en place, qui comporte les phases suivante, à savoir: évaporation, infiltration, et, ces deux phénomènes combinés.

L'objectif de ces essais est de développer une loi teneur en eau-temps pendant le séchage des sédiments avant leur traitement. Les deux phénomènes; évaporations et infiltrations ont été étudiés.

Les éprouvettes sont soumises à des conditions climatiques constantes en température et en pression. Les essais ont été réalisés dans une salle climatisée à $20^{\circ} \mathrm{C}$ et à $\mathrm{P}=\mathrm{Patm}$.

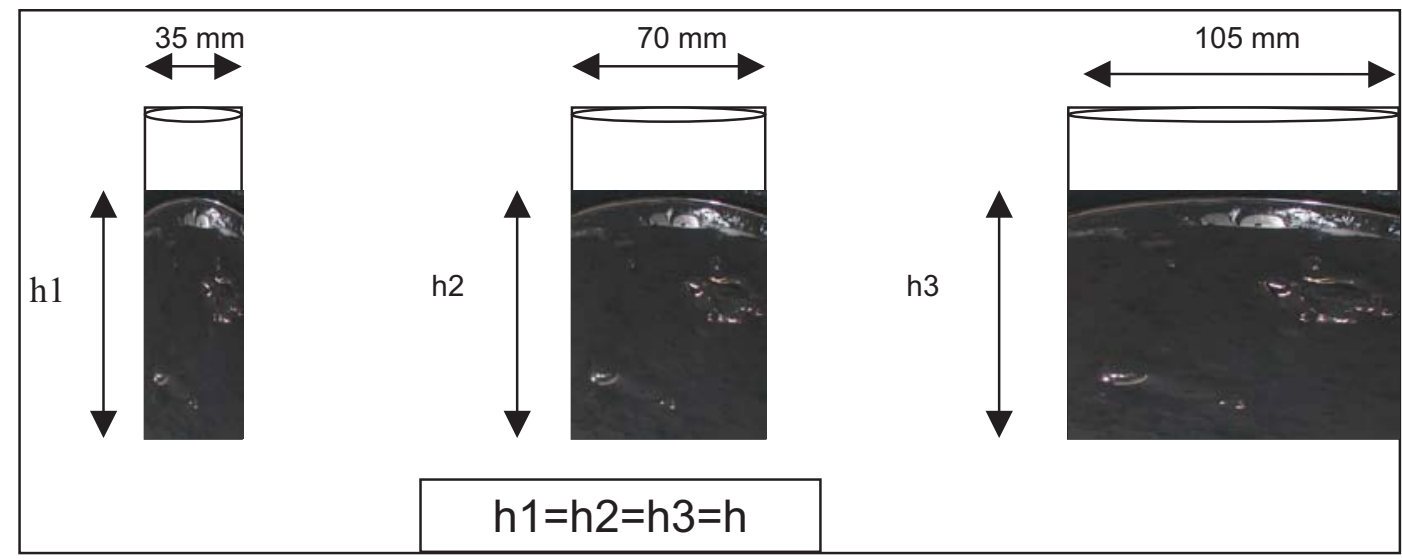

Figure 1. Essais d'évaporation - infiltration au laboratoire. 


\section{Discussions et résultats}

\subsection{Caractérisation des sédiments de dragage}

La teneur en eau initiale est d'environ 181\%. Elle est caractéristique des sédiments de dragage et dépend essentiellement de l'origine des sédiments et du type d'équipement utilisé pour le dragage (ALZIEU, 1999 ; DUAN, 2008). La distribution granulométrique des grains, obtenus par diffraction laser, a confirmé que les sédiments sont principalement composés de limon (tableau 1).

\section{Tableau 1. Caractéristiques physiques des sédiments de dragage.}

\begin{tabular}{ll}
\hline Paramètres & Valeurs \\
\hline Teneur en eau (\%) & 181 \\
$\%<2 \mu \mathrm{m}($ argile) & 8,5 \\
$2 \mu \mathrm{m}<\%<63 \mu \mathrm{m}$ (limon) & 80 \\
$63 \mu \mathrm{m}<\%$ (sable) & 2,5 \\
$\rho_{\mathrm{s}}\left(\mathrm{kg} / \mathrm{m}^{3}\right)$ & 2440 \\
Bleu de méthylène $\mathrm{V}_{\mathrm{BS}}$ & 3,25 \\
Matière organique $(\%)$ & 6,8 \\
$\mathrm{~W}_{\mathrm{L}}(\%)$ & 105,8 \\
$\mathrm{~W}_{\mathrm{P}}(\%)$ & 55,4 \\
$\mathrm{IP}(\%)$ & 50,3 \\
\hline
\end{tabular}

La quantité de matière organique contenue dans les sédiments a été évaluée par la méthode thermique, selon la norme XP P94 047. Elle est proportionnelle au carbone organique contenu dans les sédiments (MUSTIN, 1987). La limite de liquidité ( $\left.\mathrm{W}_{\mathrm{L}}\right)$ des sédiments, qui représente la limite entre l'état liquide et l'état plastique telle que mesurée par la coupelle de Casagrande, est d'environ $105 \%$, et la limite de plasticité $\left(\mathrm{W}_{\mathrm{P}}\right)$, qui représente la limite entre les l'état plastique et l'état solide, mesurés par la méthode de rouleau, est d'environ 55\%. Selon l'indice de plasticité (IP) les sédiments possèdent des caractéristiques similaires à des argiles très cohérentes peu perméables.

\subsection{Impacts et facteurs de séchage}

Les sédiments possèdent un potentiel d'infiltration très faible au total.

Au début du séchage la pente est plus importante (figure 2).

Elle atteint $0,31 \%$ par jour sur les 10 premiers jours. Cette valeur diminuera jusqu'une valeur de $0,021 \%$ par jour pour le reste du séchage.

Dans la figure 3 on présente l'évolution du séchage sur les trois échantillons soumis à l'évaporation et l'infiltration (figure 1.b).

$\mathrm{Au}$ bout de la première semaine, l'infiltration s'arrête, ceci revient au colmatage des fines entres elles. Cependant, au bout de 10 jours de séchage, la pente reste constante.

Donc le potentiel d'évaporation est constant étant donné qu'il ne dépend que des conditions d'essai. 


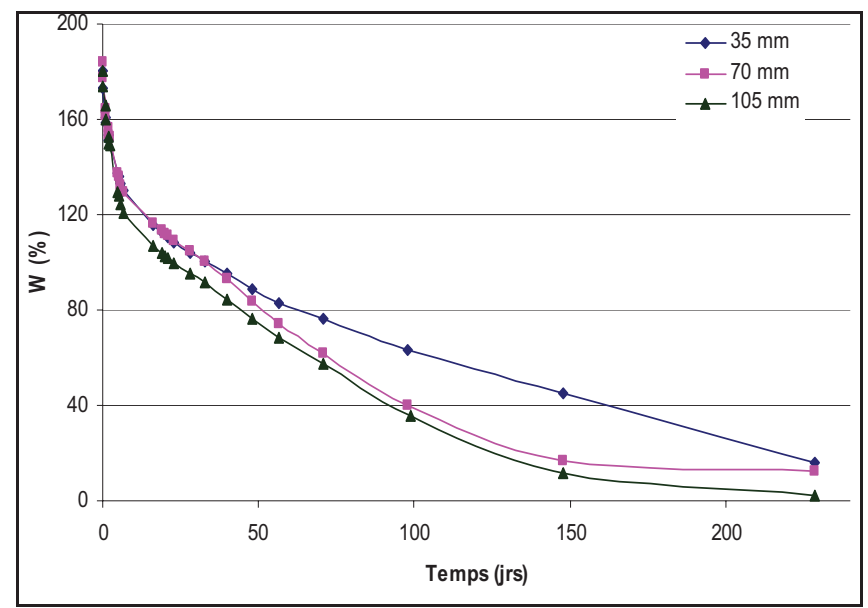

Figure 2. Séchage des sédiments.

\section{Conclusion}

L'emploi des sédiments de dragage comme matériaux en génie civil et en particulier en matériaux routier présente une alternative très attendue. D'une part l'utilisation des matériaux naturels est de plus en plus limitée et l'ouverture de nouvelles carrières est très contrôlée. En plus, une telle valorisation optimisera la gestion des stocks importants de sédiments générés chaque année. Cependant, le matériau reste à traiter et en, aucun cas n'est utilisable en l'état comme matériau routier. L'étude du séchage naturel a permis de connaitre l'évolution de la teneur en eau dans les sédiments. Cela nous permettra donc d'optimiser la préparation des sédiments à la valorisation.

\section{Références}

ALZIEU C. (1999). Dragages et environnement. Etat des connaissances. Ifremer Publications. $220 \mathrm{p}$.

DUAN Z. (2008). Caractérisation, stabilisation et solidification de sédiments fins marins. Thèse de doctorat, Université de Caen, 144p.

DUBOIS V. (2006). Etude du comportement physico-mécanique et caractérisation environnementale des sédiments marins - Valorisation en technique routière. Thèse de doctorat, ENSM de Douai-Université d'Artois, 189 p.

HAMER K., KARIUS V. (2002). Brick production with dredged harbour sediments. An industrial-scale experiment. Waste Management 22, pp 521-530

MUSTIN M. (1987). Le compost. Editions François Dubusc, 954 p.

TRAN N.T (2009). Valorisation de sédiments marins et fluviaux en technique routière. Thèse de doctorat, ENSM de Douai-Université d'Artois, 159 p.

XP P94 047. (Décembre 1998). Sols : reconnaissance et essais - détermination de la teneur en matière organique - méthode par calcination.

ZENTAR R., ABRIAK N.-E., DUBOIS V. (2008). Effects of salts and organic matter on Atterberg limits of dredged marine sediments. Applied Clay Science, pp 391-397. 